\title{
ANÁliSE DE ESTUdOS COMPUTACIONAIS DE FÁRMACOS PROMISSORES ANTI SARS-COV-2: UMA REVISÃO INTEGRATIVA
}

\section{ANALYSIS OF COMPUTATIONAL STUDIES OF PROMISING DRUG ANTI SARS- COV-2: AN INTEGRATIVE REVIEW}

\author{
Alessandra Ribeiro M. da Silva ${ }^{1}$, Allan N. dos Santos ${ }^{1}$, Agnaldo S. Carneiroํ. \\ 1 Universidade Federal do Pará \\ E-mail para correspondência: agnaldosc@ufpa.br \\ Submetido em: 30/09/2021 e aprovado em: 10/01/2022
}

\begin{abstract}
RESUMO
Introdução: em razão da recente doença, Covid-19, que se espalhou rapidamente tornando-se uma emergência sanitária a nível global, foi necessário a mobilização e esforços de cientistas e autoridades sanitárias de todo mundo, no sentido de estabelecerem medidas de segurança e iniciarem uma busca sistemática de possíveis tratamentos. Métodos: esta revisão analisou estudos de sreening virtual e docking molecular com antivirais pré-selecionados com a finalidade de encontrar evidências, por meio de estudos computacionais, de fármacos que podem ser promissores contra o SARS-COV-2. Resultados e discussão: com o auxílio da plataforma Rayyan (Intelligente Syustematic Review), estão descritos neste trabalho os resultados obtidos dos estudos para o tratamento do novo coronavírus. Conclusão: entretanto, vale ressaltar a necessidade de mais evidências computacionais e estudos clínicos controlados para encontrar uma possível cura.
\end{abstract}

Palavras-chave: Coronavírus, reaproveitamento de drogas, docking molecular

\begin{abstract}
Introduction: due to the recent disease, Covid-19, which quickly spread and became a global health emergency, it was necessary the mobilization and efforts of scientists and health authorities from around the world, to establish safety measures and start a search systematic approach to possible treatments. Methods: this review analyzed virtual sreening and molecular docking studies with pre-selected antivirals to find evidence, through computational studies, of drugs that may be promising against SARS-COV-2. Results and discussion: with the aid of the Rayyan platform (Intelligente Syustematic Review), the results obtained from the studies for the treatment of the new coronavirus are described in this work. Conclusion: however, it is worth emphasizing the need for more computational evidence and controlled clinical studies to find a possible cure.
\end{abstract}

Keywords: Coronavirus, drug repurposing, molecular docking. 


\section{INTRODUÇÃO}

Em face do cenário atual, o surto de coronavírus (SARS-COV-2), ocasionador da Covid19, gerou um temor global. Muito se tem discutido, acerca da doença que se espalhou pelo mundo rapidamente causando diversos impactos. Por conseguinte, motivou uma corrida de pesquisadores para elaborar planos estratégicos para controlar a pandemia ${ }^{(1)}$.

Os coronavírus (CoVs) foram identificados pela primeira vez em 1960 e foram classificados como membros da família Coronaviridae ${ }^{(1)}$. Essa família é conhecida por constituir uma gama de vírus que possuem como material genético o $\mathrm{RNA}^{(1)}$. O coronavírus batizado como SARS-CoV-2 começou a circular no final de dezembro de 2019 em Wuhan, capital da província de Hubei da China Central, se espalhou rapidamente pelo mundo ${ }^{(3,4)}$.

Desde o surgimento da doença, alguns casos de reinfecção com variantes filogeneticamente distintas de SARS-CoV-2 foram relatados. Esses casos de reinfecção podem ser a consequência de uma imunidade protetora limitada e transitória induzida pela primoinfecção ou podem refletir a capacidade do vírus reinfectante de escapar das respostas imunológicas anteriores ${ }^{(5,6,7,8)}$.

Os sintomas comuns de uma pessoa infectada com coronavírus incluem sintomas respiratórios, febre, tosse, falta de ar e dispneia. Em casos mais graves, a infecção pode causar pneumonia, síndrome respiratória aguda grave, insuficiência renal e até a morte ${ }^{(9,10,11)}$. Até o momento, nenhum medicamento específico foi aprovado para o tratamento do coronavírus humano. Portanto, os CoVs são considerados uma espécie de vírus, cujo surto representa uma grande ameaça para os seres humanos ${ }^{(11)}$.

No que se refere ao desenvolvimento de medicamentos para o tratamento da SARS-COV2, a maneira mais rápida e de menor custo é encontrar moléculas potenciais disponíveis no mercado de medicamentos ${ }^{(7,8,12)}$.

Estudos relacionados à reutilização de drogas para o tratamento de COVID-19 têm como objetivo identificar inibidores da protease principal (M ${ }^{\text {pro }}$ ) da SARS-COV-2, que é essencial para a replicação e transcrição viral e da proteína de pico do SARS-COV-2, uma glicoproteína que dá aos coronavírus uma aparência semelhante à de uma coroa formando pontas em sua superfície ${ }^{(12)}$. A proteína de pico CoV se liga a uma membrana da célula hospedeira por meio de uma interação mediada por receptor que permite a entrada na célula hospedeira ${ }^{(8,9)}$.

O uso de ferramentas in sílico para descoberta de fármacos tem sido amplamente adotado nos dias de hoje, merecendo especial atenção a modelagem molecular, a qual pode fornecer as 
principais interações entre os ligantes e o alvo biológico desejado, com o objetivo de esclarecer o modo de ação desses agentes químicos ${ }^{(8,9,13)}$.

Neste sentido, o presente trabalho busca efetuar um levantamento bibliográfico de estudos computacionais de fármacos antivirais, que já circulam no mercado no tratamento de infecções virais como: gripe comum-influenza, hepatite C, HIV e ebola. Este método de baixo custo e de alta precisão tem o intuito de verificar quais moléculas apresentam maior interação com o alvo receptor, indicando um possível fármaco que possa ser incluído no procedimento de "reposicionamento", bem como, uma vez estabelecidos o comportamento químico fármacoreceptor, propor possíveis modificações moleculares que conduzam a um aumento da potência dessas moléculas frente ao novo coronavírus.

\section{SARS-COV-2}

Os CoVs são vírus envelopados com genoma de RNA positivo, pertencentes à família Coronaviridae da ordem Nidovirales, que são divididos em quatro gêneros $(\alpha, \beta, \gamma$ e $\delta)$. O SARS-CoV-2 pertence ao gênero $\beta^{(5,6)}$.

Em todo o mundo, existem múltiplas variantes da síndrome respiratória aguda grave coronavírus 2 (SARS-CoV-2), o vírus que causa a doença Covid-19 $9^{(1,3,4,10)}$. Segundo a Organização Mundial da Saúde (OMS), em 15 de junho de 2021, foi registrada a marca de mais de 175 milhões de casos confirmados e mais de 3,80 milhões de óbitos mundialmente acometidos pela pandemia ${ }^{(8)}$.

A OMS passou a identificar as variantes com maior potencial de infecção como: Alfa (B.1.1.17) - Reino Unido, Beta (B.1.351) - África do Sul, Gama (P.1 ou P.2) - Brasil e Delta (B.1.617.2) - Índia.

A estrutura viral é formada principalmente pelas proteínas estrutural Spike (S) ou proteína de Pico, membrana (M), envelope (E) e nucleocapsídeo $(\mathrm{N})$ como ilustrado na figura 1. As proteínas S, M e E estão embutidas no envelope viral, que é uma bicamada lipídica derivada da membrana da célula hospedeira. A proteína $\mathrm{N}$, por outro lado, interage com o RNA viral no núcleo do vírion $^{(1)}$. 
Figura 1: Ilustração da estrutura esquemática do SARS-COV-2

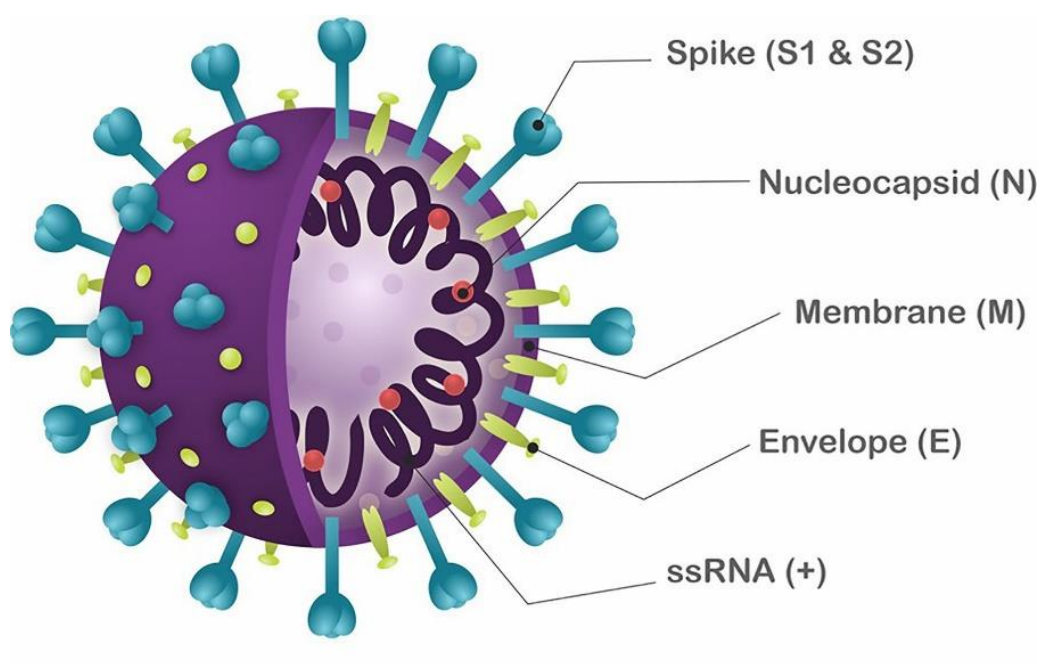

SARS-CoV-2

Fonte: Santos et al. 2020.

\section{MEDICAMENTOS}

Remdesivir

Remdesivir é um éster carboxílico resultante da condensação formal do grupo carboxi de $\mathrm{N}-[(\mathrm{S})-\{[(2 \mathrm{R}, 3 \mathrm{~S}, 4 \mathrm{R}, 5 \mathrm{R})-5-$ (4-aminopirrolo [2,1-f] [1, 2,4] triazin-7-il) -5-ciano-3,4-dihidroxitetra-hidrofuran-2-il] metoxi\} (fenoxi) fosforil] -L-alanina com o grupo hidroxi de 2etilbutan-1-ol. Sua estrutura química $\left(\mathrm{C}_{27} \mathrm{H}_{35} \mathrm{~N}_{6} \mathrm{O}_{8} \mathrm{P}\right)$ é representada na figura $2 \mathrm{~A}^{(15)}$.

Segundo Jorgensen e colaboradores (2020) Remdesivir é um pró-fármaco fosforamidato de um análogo de nucleotídeo que é metabolizado intracelularmente em um análogo de trifosfato de adenosina que inibe RNA polimerases virais. Ele inibe a replicação viral competindo com nucleotídeos endógenos envolvidos na replicação de RNA viral via RNA polimerase de que é desenvolvido a partir da protease RNA-dependente $(\operatorname{RdRp})^{(16)}$. Remdesivir tem atividade de amplo espectro contra membros de várias famílias de vírus, incluindo filovírus (por exemplo, Ebola) e coronavírus (por exemplo, SARS-CoV e coronavírus da síndrome respiratória do Oriente Médio [MERS-CoV]) e mostrou eficácia profilática e terapêutica em modelos não clínicos desses coronavírus ${ }^{(7)}$.

\section{Ribavirina}

A ribavirina é um 1-ribosiltriazol que é o derivado 1-ribofuranosil de 1,2,4-triazol-3carboxamida. Um análogo sintético da guanosina, é um inibidor da polimerase do HCV e possui 
um amplo espectro de atividade contra vírus de DNA e RNA. Sua estrutura química $\left(\mathrm{C}_{8} \mathrm{H}_{12} \mathrm{~N}_{4} \mathrm{O}_{5}\right)$ é representada na figura $2 \mathrm{~B}^{(15)}$.

$\mathrm{O}$ agente antiviral ribavirina (1-3-D-ribofuranosil-lH-1,2,4-triazol-3-carboxamida) foi aprovado pelo Food and Drug Administration em 1986 para uso como um aerossol para crianças com infecções graves devido ao vírus sincicial respiratório ${ }^{(17)}$. Este agente antiviral tem atividade inibitória contra um amplo espectro de vírus de DNA e RNA. A ribavirina é responsável por inibir proteína não estrutural RdRp que é responsável pela replicação do RNA da proteína estrutural ${ }^{(17)}$. Esta atividade antiviral é devido à semelhança deste composto em nucleosídeos. A ribavirina demonstrou eficácia clínica contra os vírus influenza A e B, vírus sincicial respiratório (RSV), e infecções por vírus parainfluenza ${ }^{(18)}$.

Favipiravir

O favipiravir é um derivado da pirazinocarboxamida com atividade contra vírus de RNA. O favipiravir (figura 2C) é convertido no derivado de ribofuranosiltrifosfato pelas enzimas do hospedeiro e inibe seletivamente a RNA polimerase dependente de RNA viral da influenza $^{(15)}$.

O trifosfato de favipiravir é um análogo do nucleosídeo de purina, que atua como um inibidor competitivo da RNA polimerase dependente de RNA (RdRp). Tem atividade contra influenza A e $\mathrm{B}^{(19)}$.

Favipiravir já foi licenciado no Japão para uso contra vírus influenza emergentes resistentes a outros antivirais. Embora, o favipiravir tenha sido desenvolvido inicialmente para ser eficaz contra infecções do vírus influenza, também se mostrou ativo contra uma ampla gama de vírus de RNA de fita positiva como o SARS-COV-2, incluindo muitos para os quais não há opções de tratamento licenciadas atualmente ${ }^{(20)}$.

\section{Lopinavir / Ritonavir}

O Lopinavir, cuja estrutura química é representada na figura $2 \mathrm{D}, \mathrm{C}_{37} \mathrm{H}_{48} \mathrm{~N}_{4} \mathrm{O}_{5}$, é uma diamida de ácido dicarboxílico. O Ritonavir apresenta em sua estrutura química (figura 2E), $\mathrm{C}_{37} \mathrm{H}_{48} \mathrm{~N}_{6} \mathrm{O}_{5} \mathrm{~S}_{2}$, é um derivado da L-valina ${ }^{(15)}$.

A combinação de drogas lopinavir/ritonavir foi sugerida como um tratamento antiviral para COVID-19. Lopinavir é um tipo de inibidor de protease que tem sido amplamente aplicado no tratamento clínico para infecção por HIV-1, que é combinado com ritonavir para aumentar 
sua meia-vida plasmática. Atualmente, o Lopinavir/Ritonavir é um dos medicamentos usados, de segunda linha, para o tratamento do $\mathrm{HIV}^{(20)}$.

Lopinavir interage com a protease do HIV causando desregulação da estrutura de proteínas funcionais no núcleo do vírus, contribuindo para a geração de partículas não infecciosas de vírus, por sua vez, alcançando a inibição de Replicação do HIV ${ }^{(22)}$. Além disso, o lopinavir tem atividade inibitória in vitro contra o coronavírus SARS-CoV, SARS-CoV-2 e síndrome respiratória do Oriente Médio (MERS) ${ }^{(21)}$.

A coadministração com ritonavir (um inibidor do citocromo A isoenzima P450 3A) inibe o metabolismo do lopinavir, aumentando significativamente as concentrações plasmáticas da droga e proporcionando alto níveis consistentes de lopinavir. Assim, uma coformulação de lopinavir e ritonavir (lopinavir / ritonavir) foi desenvolvida ${ }^{(23)}$.

O mecanismo de infecção por SARS-CoV-2 depende do processamento da poliproteína viral, um evento catalisado pela proteinase principal $\mathrm{M}^{\text {pro }}$ (também conhecida como $3 \mathrm{CL}^{\text {pro }}$ ). A $M^{\text {pro }}$ é uma enzima chave dos coronavírus e tem um papel fundamental na mediação da replicação e transcrição viral, tornando-a um alvo de droga atraente para o SARS-CoV- $2^{(5)}$. Como a $\mathrm{M}^{\text {pro }}$ é única no vírus e não é encontrada nas células hospedeiras, esta proteína é um alvo importante para o desenvolvimento de antivirais contra infecções por coronavírus.

Figura 2: Moléculas de fármacos antivirais analisadas

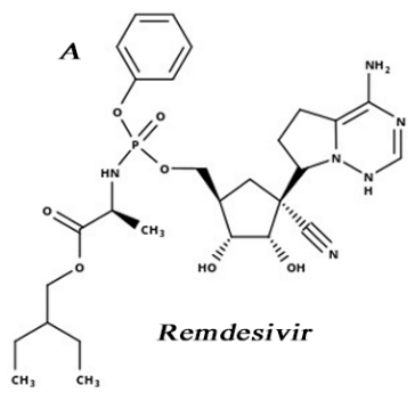

B<smiles>C[C@H]1[C@@H](CO)O[C@H](n2cnc(C(N)O)n2)[C@H]1O</smiles>

$C$<smiles>NC(=O)c1nc(F)cnc1O</smiles>

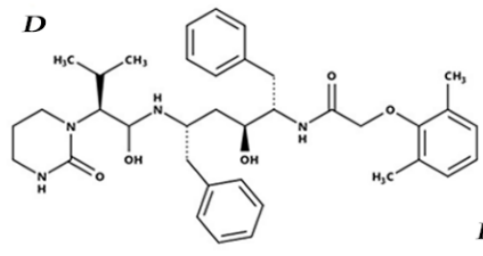

Favipiravir

Lopinavir

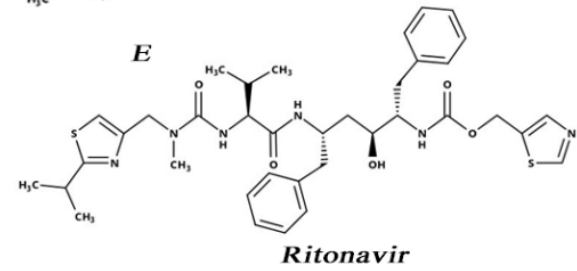

Legenda: (A) Estrutura química do Remdesivir; (B) Estrutura química do Ribavirina; (C) Estrutura química do Favipiravir; (D) Estrutura química do Lopinavir / (E) ritonavir. Fonte: autores, 2021. 


\section{ANÁLISE IN SÍLICO}

As análises in sílico representam uma série de métodos computacionais (screening Virtual, Docagem Molecular e Dinâmica Molecular) que vem chamando atenção da indústria farmacêutica, por apresentarem rapidez em sua execução, baixo custo e redução da utilização de animais em atividades experimentais. Através da utilização desses métodos a identificação de moléculas promissoras tornou-se mais eficiente, alocando os recursos para estudos in vitro e in vivo de moléculas com maior probabilidade de serem utilizadas como fármacos ${ }^{(1)}$.

\section{SREENING VIRTUAL}

A busca virtual consiste em pré-selecionar os compostos com o auxílio do computador a partir de bancos de dados virtuais com um grande número de moléculas. Desta forma, apenas os compostos pré-selecionados são submetidos aos testes de atividade in vitro. Esta pré-seleção é feita prevendo-se, virtualmente, a atividade biológica de interesse por meio de diferentes abordagens LBDD (do inglês, structure based drug design) e SBDD (do inglês, ligand based drug design). Como vantagens desta estratégia têm-se que os compostos estudados não precisam, necessariamente, existir fisicamente e que neste "teste virtual", in silico, não há consumo de material ${ }^{(24)}$.

\section{DOCKING MOLECULAR}

O docking molecular inicialmente foi projetado para prever e reproduzir complexos. Entretanto, passou a ser uma ferramenta de suma importância, tendo em vista que auxilia na otimização e descoberta de novos medicamentos ${ }^{(3)}$.

A docagem é um método que prevê a orientação preferencial de uma molécula frente a um sistema biológico conhecido. O conhecimento da orientação preferida, por sua vez pode ser utilizado para prever a força de associação ou a afinidade de ligação entre fármaco e o receptor, gerando um valor chamado de scoring ${ }^{(25)}$.

O método de docking molecular desempenha um papel fundamental no processo de design e descoberta de drogas. O principal objetivo do docking molecular é simular computacionalmente o processo de identificação molecular de forma que a energia livre do sistema seja minimizada ${ }^{(25)}$. 


\section{POTENCIAIS ALVOS}

Entre os alvos baseados em vírus, a importância da proteína estrutural spike (S) é notável, devido ao seu papel principal na entrada do SARS-CoV-2, por meio da interação com o receptor ACE2, no hospedeiro. Neste contexto, também é de especial relevância para a replicação do vírus as proteases (3CL ${ }^{\text {pro }}$ e $\mathrm{PL}^{\text {pro }}$ ), a RNA polimerase dependente de RNA (RdRp) e a helicase $^{(4)}$.

\section{MÉTODOS}

Fontes de Informação e Descritores de pesquisa

As buscas na literatura foram realizadas nas bases de dados Medline (via Pubmed), Embase, The Cochrane Library, Scielo, Google acadêmico. Foram utilizados descritores específicos "SARS-COV-2", "Studies in sílico", "Antivirals", "Molecular docking SARSCOV-2", "Covid 19" todos no idioma em inglês. A busca foi conduzida no dia 01 de maio de 2020, com última atualização feita em 10 de maio de 2020 com as palavras chave relacionadas ao assunto principal "Coronavírus", "Reaproveitamento de drogas", “Docking molecular" através do descritor em ciências da saúde DESC. Os operadores booleanos não foram aplicados na pesquisa.

Critérios de inclusão

Estudos que avaliam a ação de medicamentos antivirais no tratamento de indivíduos com infecções respiratórias virais (MERS-CoV, SARS-CoV e SARS-CoV-2) foram considerados elegíveis para inclusão na presente revisão. Não foram aplicados filtros relacionados a data, ou status de publicação. Os tipos de estudos incluídos foram: artigos publicados e relato de caso com abordagens computacionais de Screening virtual e docking molecular.

Critérios de exclusão

Foram excluídos estudos que não analisaram antivirais, ou seja, produtos naturais ou extratos de plantas medicinais não foram incluídos. Ensaios pré-clínicos, in vitro e in vivo também não se encaixam no padrão de seleção. Foram também excluídos estudos que discordam das informações repassadas pelo órgão majoritário da saúde "Organização Mundial da Saúde" (OMS). 
Tratamento dos dados

Para a realização da revisão atual, criou-se uma pasta no aplicativo de seleção Rayyan (Intelligente Syustematic Review) $^{(2)}$ com o intuito de auxiliar na seleção de estudos e na pesquisa. A busca de estudos ocorreu através dos principais descritores e base de dados. A seleção de estudos, foi realizada de maneira independente por dois revisores respeitando-se os critérios de inclusão e exclusão de acordo com a leitura do título e resumo. Posteriormente, Cada um dos autores excluiu o estudo ou incluiu o estudo no aplicativo Rayyan, e aqueles selecionados no aplicativo foram avaliados de acordo com o texto completo e, encaminhados para a extração de dados.

A finalidade desta revisão é identificar os fármacos mais promissores contra a covid-19, através de estudos in sílico. Assim, discutindo seus alvos, informações fisiopatológicas e mecanismos de ação farmacológica. Por isso, abordou-se cinco medicamentos: Remdesivi, Ribavirim, Favipiravir, Lopinavir/ritonavir que foram os antivirais mais citados nos estudos selecionados.

\section{RESULTADOS E DISCUSSÃO}

No quadro 01 é possível verificar algumas informações dos estudos selecionados. Ao todo foram selecionados 32 estudos, em que 24 foram excluídos após leitura completa, ou seja, 8 estudos foram compatíveis com as exigências. Contabilizando apenas $25 \%$ dos estudos admitidos. 
Quadro 01: Estudos selecionados no aplicativo Rayyan (Intelligente Syustematic Review)

\begin{tabular}{|c|c|c|c|}
\hline Título & Autores & $\begin{array}{c}\text { Revista e ano da } \\
\text { publicação }\end{array}$ & Resultados \\
\hline $\begin{array}{l}\text { A search for } \\
\text { medications to treat } \\
\text { COVID-19 via in silico } \\
\text { molecular docking } \\
\text { models of the SARS- } \\
\text { CoV-2 spike } \\
\text { glycoprotein and } 3 \mathrm{CL} \\
\text { protease }\end{array}$ & $\begin{array}{l}\text { Hall, Donald C.; } \\
\text { Ji, Hai-Feng; }\end{array}$ & $\begin{array}{l}\text { Travel Medicine and } \\
\text { Infectious Disease - } \\
\text { Volume 35, Issue 0, } \\
\text { pp. 101646 - } \\
\text { published 2020-01-01 }\end{array}$ & $\begin{array}{l}\text { Os compostos de sucesso relatados } \\
\text { apresentam potencial para inibir a proteína } \\
\text { spike SARS-CoV-2 e a protease principal } \\
\text { 3CL }{ }^{\text {PRO }} \text {, mas não é garantido que tenham } \\
\text { qualquer atividade; Remdesivir é um } \\
\text { composto antiviral em estágios experimentais } \\
\text { que mostrou atividade contra o SARS- } \\
\text { coronavírus, Ebolavírus e, possivelmente, o } \\
\text { SARS-CoV-2. }\end{array}$ \\
\hline $\begin{array}{l}\text { Analysis of therapeutic } \\
\text { targets for SARS-CoV- } \\
2 \text { and discovery of } \\
\text { potential drugs by } \\
\text { computational methods }\end{array}$ & $\begin{array}{l}\text { Wu, Canrong; Liu, } \\
\text { Yang; Yang, } \\
\text { Yueying; Zhang, } \\
\text { Peng; Zhong, Wu; } \\
\text { Wang, Yali; Wang, } \\
\text { Qiqi; Xu, Yang; Li, } \\
\text { Mingxue; Li, } \\
\text { Xingzhou; Zheng, } \\
\text { Mengzhu; Chen, } \\
\text { Lixia; Li, Hua } \\
\end{array}$ & $\begin{array}{l}\text { Acta Pharmaceutica } \\
\text { Sinica B - Volume } \\
\text { 10, Issue 5, pp. 766- } \\
788 \text { - published 2020- } \\
\text { 01-01 }\end{array}$ & $\begin{array}{l}\text { Os resultados de todo o artigo são baseados } \\
\text { em triagem virtual por computador, } \\
\text { modelagem por homologia e docking } \\
\text { molecular. Medicamentos antivírus } \\
\text { (ribavirina, valganciclovir e timidina), As } \\
\text { ações farmacológicas originais dessas drogas } \\
\text { podem ser úteis para o tratamento da } \\
\text { pneumonia por infecção viral. }\end{array}$ \\
\hline $\begin{array}{l}\text { Computational } \\
\text { screening of } \\
\text { antagonists against the } \\
\text { SARS-CoV-2 } \\
\text { (COVID-19) } \\
\text { coronavirus by } \\
\text { molecular docking }\end{array}$ & $\begin{array}{l}\text { Yu, Ran; Chen, } \\
\text { Liang; Lan, Rong; } \\
\text { Shen, Rong; Li, } \\
\text { Peng; }\end{array}$ & $\begin{array}{l}\text { International Journal } \\
\text { of Antimicrobial } \\
\text { Agents - Volume 56, } \\
\text { Issue 2, pp. 106012 - } \\
\text { published 2020-01-01 }\end{array}$ & $\begin{array}{l}\text { O modelo da protease principal COVID-19 O } \\
\text { método de triagem é restrito ao docking } \\
\text { molecular, e simulação de dinâmica molecular } \\
\text { não foi realizada. }\end{array}$ \\
\hline $\begin{array}{l}\text { Drug binding dynamics } \\
\text { of the dimeric SARS- } \\
\text { CoV-2 main protease, } \\
\text { determined by } \\
\text { molecular dynamics } \\
\text { simulation }\end{array}$ & $\begin{array}{l}\text { Komatsu, Teruhisa } \\
\text { S.; Okimoto, } \\
\text { Noriaki; Koyama, } \\
\text { Yohei M.; Hirano, } \\
\text { Yoshinori; } \\
\text { Morimoto, Gentaro; } \\
\text { Ohno, Yousuke; } \\
\text { Taiji, Makoto }\end{array}$ & $\begin{array}{l}\text { Scientific Reports - } \\
\text { Volume 10, Issue 1, } \\
\text { pp. } 16986 \text { - publicado } \\
2020-01-01\end{array}$ & $\begin{array}{l}\text { Neste trabalho realizou-se simulação de } \\
\text { dinâmica molecular e docking molecular Os } \\
\text { resultados revelaram uma grande variação nas } \\
\text { formas dos locais de ligação e nas posições de } \\
\text { ligação dos ligantes. Coletivamente, esses } \\
\text { resultados indicam a importância da } \\
\text { amostragem dinâmica de complexos proteína- } \\
\text { ligante e sugerem as possibilidades de } \\
\text { otimizações de drogas adicionais. }\end{array}$ \\
\hline $\begin{array}{l}\text { Identification of } \\
\text { Potential Inhibitors of } \\
\text { Severe Acute } \\
\text { Respiratory Syndrome- } \\
\text { Related Coronavirus } 2 \\
\text { (SARS-CoV-2) Main } \\
\text { Protease from Non- } \\
\text { Natural and Natural } \\
\text { Sources: A Molecular } \\
\text { Docking Study. } \\
\end{array}$ & $\begin{array}{l}\text { Santos-Filho, } \\
\text { Osvaldo A }\end{array}$ & $\begin{array}{l}\text { Journal of the } \\
\text { Brazilian Chemical } \\
\text { Society\} - Volume } \\
\text { 31, Issue 0, pp. } 2638 \\
\text { - 2643 - published } \\
\text { 2020-12-01 }\end{array}$ & $\begin{array}{l}\text { Utilizou-se a estruturta cristalográfica do } \\
\text { SARS-CoV-2 como receptor } \\
\text { biomacromolecular em simulações de docking } \\
\text { molecular. Os resultados mostraram que dois } \\
\text { compostos não naturais, danoprevir e } \\
\text { lopinavir produziram fortes interações com o } \\
\text { sítio de ligação do inibidor da protease } \\
\text { principal SARS-CoV-2. }\end{array}$ \\
\hline $\begin{array}{l}\text { In silico prediction of } \\
\text { potential inhibitors for } \\
\text { the main protease of } \\
\text { SARS-CoV-2 using } \\
\text { molecular docking and } \\
\text { dynamics simulation } \\
\text { based drug-repurposing }\end{array}$ & $\begin{array}{l}\text { Kumar, Yogesh; } \\
\text { Singh, Harvijay; } \\
\text { Patel, Chirag N }\end{array}$ & $\begin{array}{l}\text { Journal of Infection } \\
\text { and Public Health - } \\
\text { Volume 13, Issue 9, } \\
\text { pp. 1210-1223 - } \\
\text { published 2020-01-01 }\end{array}$ & $\begin{array}{l}\text { No presente estudo, realizou-se a seleção de } \\
\text { drogas através da triagem virtual baseada em } \\
\text { docking molecular. Foram utilizados } \\
\text { inibidores Lopinavir - Ritonavir, Tipranavir e } \\
\text { Raltegravir mostram a melhor interação } \\
\text { molecular com a protease principal do SARS- } \\
\text { CoV-2. }\end{array}$ \\
\hline $\begin{array}{l}\text { Modification of } \\
\text { Remdesivir as a Better } \\
\text { Inhibitor of COVID- } \\
\text { 19: A Computational } \\
\text { Docking Study } \\
\end{array}$ & $\begin{array}{l}\text { Shikder, Mita; } \\
\text { Ahmed, Kazi } \\
\text { Ahsan; Hasib, } \\
\text { Tasnin Al; Kabir, } \\
\text { Md. Lutful }\end{array}$ & $\begin{array}{c}\text { Volume } 0 \text {, Issue } 0, \\
\text { pp. - published 2020- } \\
09-01\end{array}$ & $\begin{array}{l}\text { Neste estudo a eficácia das drogas foi testada } \\
\text { por cálculos de docking molecular. Todos os } \\
\text { modificadores (exceto DF) prevêem energia } \\
\text { de ligação mais significa e interações de não } \\
\text { ligação do que o fármaco original. }\end{array}$ \\
\hline $\begin{array}{l}\text { The Molecular } \\
\text { Docking Study of } \\
\text { Potential Drug } \\
\text { Candidates Showing } \\
\text { Anti-COVID-19 } \\
\text { Activity by Exploring } \\
\text { of Therapeutic Targets } \\
\text { of SARS-CoV-2 }\end{array}$ & $\begin{array}{l}\text { Rohan R. } \\
\text { Narkhede, } \\
\text { Rameshwar S. } \\
\text { Cheke, Jaya P } \\
\text { Ambhore, Sachin } \\
\text { D. Shinde }\end{array}$ & $\begin{array}{l}\text { EJMO - Volume 4, } \\
\text { Issue 3, pp. 185-195 - } \\
\text { published 2020-01-01 }\end{array}$ & $\begin{array}{l}\text { Os experimentos de docking molecular foram } \\
\text { realizados através do Software. Os } \\
\text { medicamentos antivirais oseltamivir, ritonavir, } \\
\text { remdesivir ribavirina e favipiravir } \\
\text { apresentaram interações de alta afinidade com } \\
\text { o COVID-19 } \mathrm{M}^{\text {pro }} \text { em complexo com o } \\
\text { inibidor N3; }\end{array}$ \\
\hline
\end{tabular}

Fonte: autores, 2021. 
No experimento de Santos-Filho ${ }^{(27)}$, foi utilizado como modelo a estrutura SARS-CoV-2 $\mathrm{M}^{\text {pro }}$ em complexo com inibidor de N3 com o código PDB(6LU7) Representado na figura 3A.

Foi possível identificar, a partir da análise de docagem que a molécula de lopinavir mostrou a melhor energia de afinidade para o SARS-CoV-2 $\mathrm{M}^{\text {pro }}$ apresentando valor de afinidade igual a $-8,5 \mathrm{kcal}^{\mathrm{mol}} \mathrm{m}^{-1} \mathrm{e}$ interage com os resíduos Thr-26, Glu-166, Gln-189 enquanto que a molécula de ritonavir apresentou energia de afinidade igual a -7,9 $\mathrm{kcal} \mathrm{mol}^{-1} \mathrm{e}$ interage com os resíduos de aminoácidos Ser-46, Gln-189. Esses valores foram obtidos a partir de interações polares e hidrofóbicas entre as drogas e o alvo do SARS-COV-2, assim como uma série de ligações de hidrogênio.

Kumar e colaboradores ${ }^{(28)}$ desenvolveram um estudo de docagem molecular de drogas antivirais contra a enzima $\mathrm{M}^{\text {pro }}$ do SARS-CoV-2, onde revelou uma forte interação com alta energia de docagem e afinidades de ligação.

Foi possível identificar na região do sitio ativo do SARS-COV-2 na protease $\mathrm{M}^{\text {pro }}$, os resíduos do domínio S1', integrados por Cys145, Gly143 e Ser144, que também servem como o orifício de oxiânion. No domínio S1 está presente o resíduo de His163, já no domínio S2 estão localizados os resíduos de Glu166 e Gln189, o domínio S4 é composto por Gln189 e Pro168 (Figura 3B). A protease se liga aos resíduos específicos buscando o início da produção de proteínas estruturais para a formação do complexo de replicação.

No estudo foi possível identificar os aminoácidos que interagem no sítio ativo da enzima $\mathrm{M}^{\text {pro }}$ (Leu, Gln, Ser, Ala, Gly) junto com a dupla Cys-His que demarcou o sítio de clivagem, o estudo in sílico mostrou que a combinação de Lopinavir-Ritonavir interage com Glu 166 (formando uma forte ligação de hidrogênio), Gln 189, Leu 167, Met 165, Asp 187, Met 49, His 41, Cys 145 e Leu 141.

O Lopinavir/ritonavir apresentou afinidade de $-10,6 \mathrm{kcal} \mathrm{mol}^{-1} \mathrm{com}$ a molécula $\mathrm{M}^{\text {pro }}$ do SARS-COV-2.

A figura 3 representa as principais ligações que a molécula de Lopinavir/ritonavir desenvolve com a protease $\mathrm{M}^{\text {pro }}$ do SARS-COV-2. Foram possíveis identificar as interações com His164, Glu166, Gln192, Agr188, His41 e Cys145. 


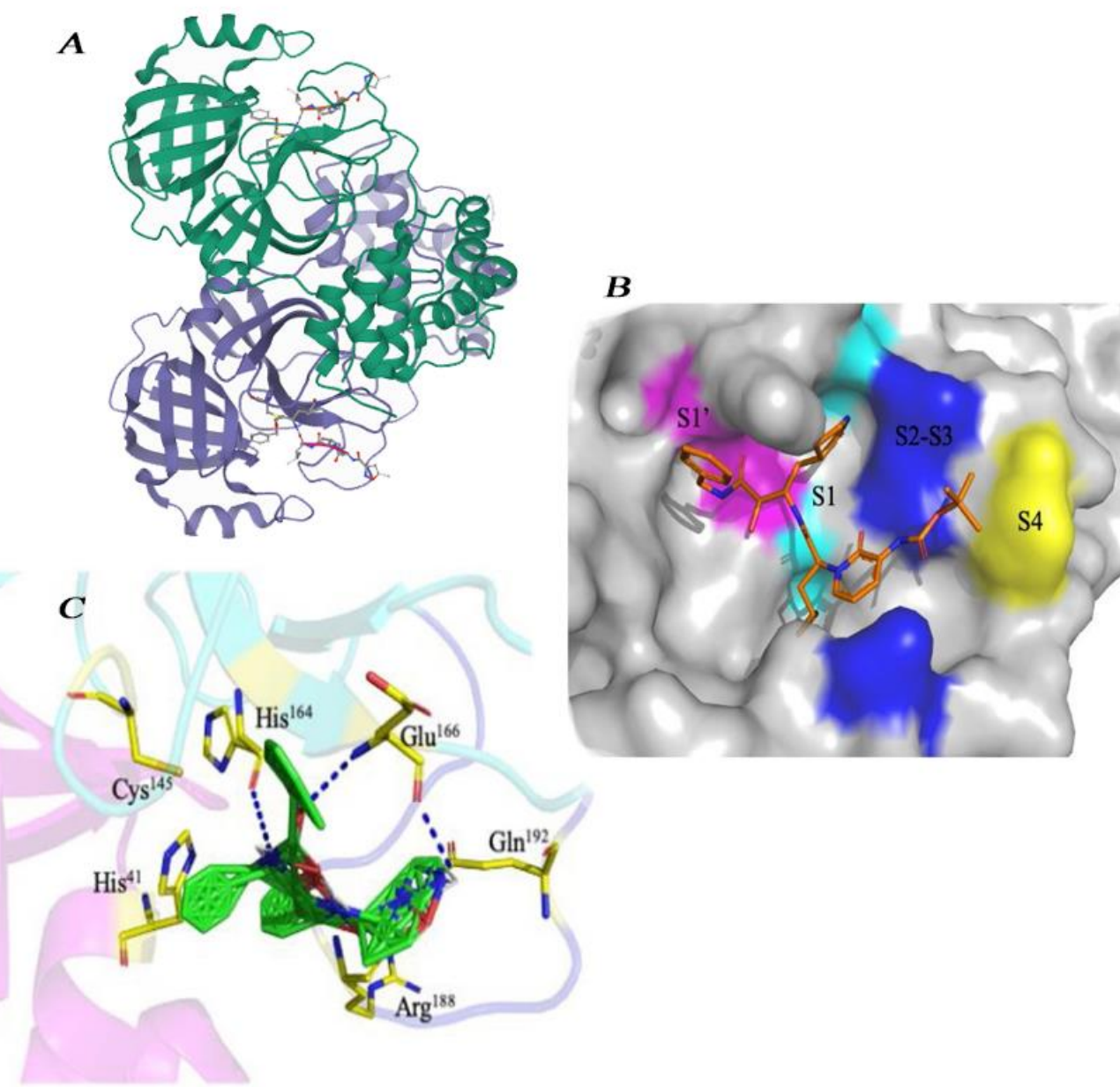

Legenda: (A) SARS-CoV-2 Mpro em complexo com inibidor de N3 (adaptado de ID do PDB: 6LU7); (B) Diferentes grupos de subsitios S1 ', S1, S2, S3 \& S4 nos subsitios de ligação ao substrato de SARS-CoV-2M Pro (PDB ID: 6Y2F); (C) Interação de docking molecular de drogas antivirais acopladas com SARS-CoV-2 Mpro. (A) Lopinavir - Ritonavir. Alta afinidade de ligação. Fonte: autores, 2021

No estudo de Narkhede e colaboradores ${ }^{(29)}$ foram obtidos resultados que revelaram fortes interações dos antivirais frente a protína $\mathrm{M}^{\text {pro }}$ e com a glicoprotéina ACE2 humana de SARSCOV-2. Neste estudo foram avaliados os antivirais Remdesivir, Ribavirina, Favipiravir, e Ritonavir. As drogas testadas computacionalmente, tiveram um alto nível de afinidade com o alvo biologico.

A molécula de Ritonavir com o SARS-COV-2 $\mathrm{M}^{\text {pro }}$ em complexo com o inibidor N3 revelou que a droga teve uma interação de alta afinidade com a cadeia da proteína: $-7,3$ kcal.mol ${ }^{-1}$ Também foi possível identificar a interação de ligação de hidrogênio entre o ritonavir e resíduos de aminoácidos da proteína. Com ênfase no oxigênio (grupo amida) que demonstrou ligação de hidrogênio significativa com THR 111. Outra ligação identificada foi com anel benzeno que apresentou interação pi-ânion e pi-pi com ASP 153 e PHE 294, respectivamente. 
No experimento com o Remdesivir no COVID-19 $\mathrm{M}^{\text {pro }}$ em complexo com o inibidor N3 foram obtidos resultados de afinidade de interação significativas na parte central da cadeia resultando em uma afinidade de $-6,5 \mathrm{kcal}^{\mathrm{m}} \mathrm{mol}^{-1}$. A principal interação entre remdesivir e a protease foi caracterizada por ligação de hidrogênio entre nitrogênio de um grupo cyno e PHE 294, e oxigênio de um anel tetrahidrofurano com GLN 110.

Os resultados alcançados a partir do encaixe da Ribavirina na molécula do SARS-COV$2 \mathrm{M}^{\text {pro }}$ em complexo com o inibidor $\mathrm{N} 3$ mostrou ligação em cadeia com uma afinidade de $-5,6$ kcal.mol ${ }^{-1}$. Também foi possível identificar significativa ligação com 4 ligações de hidrogênio entre o nitrogênio de um anel dihidrotriazol e TYR 239,

Neste trabalho foi realizado o screening virtual onde o favipiravir apresentou 6 ligações de hidrogênio e mostrou atividade promissora com o $\mathrm{M}^{\text {pro }}$ de COVID-19, com afinidade de $5,4 \mathrm{kcal}^{\mathrm{mol}}{ }^{-1}$. Apresentou forte interação com a protease. Assim, as interações de hidrogênio, são estabelecidas em dois pontos da molécula. No átomo de oxigênio ligado ao anel pirazina e no átomo de oxigênio do grupo carboxíamida, ambos com resíduos de GLN 110, THR 292 e THR 111.

No experimento de Hall $\mathrm{Jr}$ e $\mathrm{Ji}^{(8)}$ a protease principal $3 \mathrm{CL}^{\text {pro }}$ do SARS-COV-2 foi submetida a um processo de docagem a partir do software Schrodinger Docking Suits®. Estudos revelaram que a mutação da protease SARS do resíduo His162 torna a enzima inativa. Também foi possível identificar uma segunda Histidina (His41), ambos mostraram-se propícios ao desempenhar um papel importantenas interações do ligante com a estrutura da protease. Foi realizado a docagem do Remdesivir onde foi obtido uma afinidade de -7,2 $\mathrm{kcal} \mathrm{mol}^{-1}$ e ligação com os resíduos de aminoácidos Thr-26, Glu-166, Gln-189.

$\mathrm{Wu}^{(12)}$ e colaborares desenvolveram o estudo de docagem molecular a partir do software de modelagem ICM 3.7.3 a fim de verificar a precisão da modelagem por homologia. Nas análises de acoplamento da ribavirina observa-se que a molécula se ligava a protease PL ${ }^{\text {pro }}$, que é essencial para a correção de replicação do vírus, com uma baixa energia de ligação (escores $=-38,58)$. O modelo de docagem utilizado identificou a ribavirina ligada ao sítio ativo da enzima, foram observadas as ligações de hidrogênio com os resíduos Gly164, Gln270, Tyr274, Asp303, além disso, o empilhamento $\pi-\pi$ com o resíduo Tyr265. Essas ligações de hidrogênio sugerem que a Ribavirina pode ser uma terapia de inibição da protease PL ${ }^{\text {pro }}$.

Nos estudos de Shikder e colaboradores, o remdesivir apresentou uma possível interação com o canal de ligação de RNA do SARS-CoV-2. A molécula se acoplou bem ao formato da bolsa, onde formou três ligações de hidrogênio com Asn497, Arg569 e Asp684, juntamente 
com interações hidrofóbicas com Leu576, Ala685 e Tyr687. Enquanto que o favipiravir nesse estudo obteve pontuações de acoplamento irrelevantes ao alvo do SARS-COV-2.

Nos experimentos de $\mathrm{Yu}^{(30)}$ e colaboradores foi utilizado o software AutoDock Vina em todos os experimentos de encaixe onde foi possível identificar que o Remdesivir em docagem com a protease $3 \mathrm{CL}^{\text {pro }}$ formou ligações de hidrogênio com os resíduos de aminácidos GLU240, HIS-246 e PRO-108, e interações hidrofóbicas com os resíduos PRO-132 e ILE-200, e interações cátion $\pi$ com o resíduo GLN-107. As interações entre a ribavirina e a protease PL pro foram formadas ligações de hidrogênio, já com a $\mathrm{RdRp}$, a ribavirina formou ligações de hidrogênio com LEU-271, LEU-270, ASP-269 e TRP-268. Enquanto que o Remdesivir formou ligações hidrogênio com ALA-1070 e GLN-1071, e interação hidrofóbica com GLU-1072.

No estudo Komatsu ${ }^{(25)}$, as análises de ancoragem foram desenvolvidas com o auxílio do software Molecular Operating Environment (MOE). Nesse experimento foram encontrados valores de docagem para o ritonavir onde foi possível observar a sua ligação com a protease

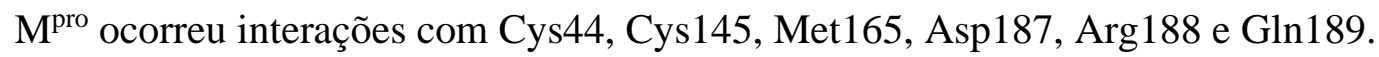

Os estudos in sílico de Hall Jr e Ji e Shikder ${ }^{(8)}$ e colaboradores indicaram que o Remdesivir é uma molécula promissora para ser usada como referência na proposta de novos fármacos contra o covid-19, uma vez que ela apresentou boa afinidade química com o receptor biológico. Neste sentido o Lopinavir, estudado por Santos-Filho e o Ritonavir estudado por Narkhede e colaboradores e por Komatsu apresentaram resultados de afinidade química semelhantes ao Remdesivir. No entanto um achado muito significativo foi proposto por Kumar e colaboradores, no qual verificou-se a possibilidade de combinação dos fármacos Lopinavir-Ritonavir, mediante a verificação de uma boa energia de ligação, abrindo o caminho para novas propostas de associação de fármacos no combate ao vírus do Sars-Cov-2.

Os resultados computacionais com a Ribavirina, executados por Wu e colaborares e Yu e colaboradores sugerem que essa molécula é a mais promissora para o combate contra o covid19, pois ela apresentou maior afinidade química com o alvo biológico. Esta descoberta possibilita a construção de novas entidades químicas a partir do perfil energético das interações estabelecidas entre o fármaco e o receptor.

\section{CONCLUSÃO}

Com o advento da pandemia de COVID-19 pesquisadores de todo o mundo buscaram alternativas medicamentosas. Com isso, estudos computacionais ofereceram possibilidades de 
reaproveitamento de drogas já existentes, o que possibilitou a otimização de tempo e acelerou o processo de busca por medicamentos eficazes.

As ferramentas na área da simulação computacional como a metodologia de docking molecular favorecem um melhor custo-benefício. Tendo em vista que, através destes ocorre a redução de gastos e redução de tempo no processo de pesquisa de futuros candidatos a fármacos.

A interrupção do SARS-COV-2 é o objetivo de vários trabalhos científicos. No entanto, se faz necessário a realização de mais estudos para elucidar, de forma segura, uma estrutura química capaz de combater a doença. Desta forma, esta revisão, surge para oferecer suporte intelectual e apresentar o empenho computacional de cientistas na busca de potenciais candidatos a fármacos.

As técnicas computacionais evidenciam a importância do uso dessas ferramentas que são aplicadas em projetos de pesquisa e desenvolvimentos de fármacos. Os estudos analisados apresentam dados que podem auxiliar na busca de medicamentos eficazes para o tratamento de complicações decorrentes da covid-19. Principalmente no que se refere a associação de fármacos, como foi verificado com a combinação Lopinavir-Ritonavir nos ensaios in sílico.

A Ribavirina mostrou-se ser uma molécula importante, do ponto de vista energético, visto que estabiliza o complexo fármaco-receptor, indicando que existe um caminho energeticamente favorável que pode inibir o vírus causador da covid-19. Com tudo, é primordial que ocorra ensaios e estudos complementares.

\section{REFERÊNCIAS BIBLIOGÁFICAS}

1. Santos IA, et al. Antivirais contra coronavírus: drogas candidatas ao tratamento da SARSCoV-2. Frontiers in Microbiology, v. 11, p. 1818, 2020.

2. Ouzzani, Mourad et al. Rayyan: um aplicativo da web e móvel para revisões sistemáticas. Revisões sistemáticas , v. 5, n. 1, pág. 1-10, 2016.

3. Khan MA et al. Comparative molecular investigation of the potential inhibitors against SARS-CoV-2 main protease: a molecular docking study. Journal of Biomolecular Structure and Dynamics, p. 1-7, 2020.

4. Gil C, et al. COVID-19: drug targets and potential treatments. Journal of medicinal chemistry, v. 63, n. 21, p. 12359-12386, 2020.

5. Jin Z, et al. Structure of M pro from SARS-CoV-2 and discovery of its inhibitors. Nature, v. 582, n. 7811, p. 289-293, 2020.

6. Naveca F, et al. Reinfecção do SARS-CoV-2 pelo novo Variant of Concern (VOC) P. 1 no Amazonas, Brasil. virológico. org, 2021. 
7. Grein J, et al. Compassionate use of remdesivir for patients with severe Covid-19. New England Journal of Medicine, v. 382, n. 24, p. 2327-2336, 2020.

8. Hall Jr DC, Ji HF. A search for medications to treat COVID-19 via in silico molecular docking models of the SARS-CoV-2 spike glycoprotein and 3CL protease. Travel medicine and infectious disease, Philadelphia, v. 35, p. 101646, 2020.

9. Ojha PK, et al. Therapeutics for COVID-19: from computation to practices - where we are, where we are heading to. Molecular diversity, v. 25, n. 1, p. 625-659, 2021.

10. Abdool K, Salim S, De Oliveira, T. New SARS-CoV-2 variants-clinical, public health, and vaccine implications. New England Journal of Medicine, 2021.

11. Babiker A, et al. The importance and challenges of identifying SARS-CoV-2 reinfections. Journal of clinical microbiology, v. 59, n. 4, 2021.

12. Wu C, et al. Analysis of therapeutic targets for SARS-CoV-2 and discovery of potential drugs by computational methods. Acta Pharmaceutica Sinica B, v. 10, n. 5, p. 766-788, 2020.

13. Faria SHDM, Teleschi JG. Computational search for drug repurposing to identify potential inhibitors against SARS-COV-2 using Molecular Docking, QTAIM and IQA methods in viral Spike protein-Human ACE2 interface. Journal of molecular structure, v. 1232, p. 130076, 2021.

14. Gajjar ND, Dhameliya TM, Shah GB. In Search of RdRp and $M^{\text {pro }}$ Inhibitors against SARS CoV-2: Molecular Docking, Molecular Dynamic Simulations and ADMET Analysis. Journal of molecular structure , p. 130488, 2021.

15. PUBCHEM. National Center for Biotechnology Information. Disponível em: https://pubchem.ncbi.nlm.nih.gov/. (Acesso em: 06/09/2021).

16. Jorgensen SCJ, Kebriaei R, Dresser LD. Remdesivir: review of pharmacology, pre-clinical data, and emerging clinical experience for COVID-19. Pharmacotherapy: The Journal of Human Pharmacology and Drug Therapy, v. 40, n. 7, p. 659-671, 2020.

17. Patterson JL, Fernandez-larsson R. Molecular mechanisms of action of ribavirin. Reviews of infectious diseases, v. 12, n. 6, p. 1139-1146, 1990.

18. Gilbert BE, Knight V. Biochemistry and clinical applications of ribavirin. Antimicrobial agents and chemotherapy, v. 30, n. 2, p. 201-205, 1986.

19. Coomes EA, Haghbayan H. Favipiravir, an antiviral for COVID-19?. Journal of Antimicrobial Chemotherapy, v. 75, n. 7, p. 2013-2014, 2020.

20. Goldhill DH, et al. The mechanism of resistance to favipiravir in influenza. Proceedings of the National Academy of Sciences, v. 115, n. 45, p. 11613-11618, 2018.

21. Horby PW, et al. Lopinavir- ritonavir in patients admitted to hospital with COVID-19 (RECOVERY): a randomised, controlled, open-label, platform trial. The Lancet, v. 396, n. 10259, p. 1345-1352, 2020. 
22. Ye XT, et al. Clinical efficacy of lopinavir/ritonavir in the treatment of Coronavirus disease 2019. Eur Rev Med Pharmacol Sci, v. 24, n. 6, p. 3390-3396, 2020.

23. Hurst M, Faulds D. Lopinavir. Drugs, v. 60, n. 6, p. 1371-1379, 2000.

24. Ferreira LG, et al. Docking molecular e estratégias de desenho de drogas baseadas em estrutura. Molecules, v. 20, n. 7, pág. 13384-13421, 2015.

25. Komatsu TS, et al. Drug binding dynamics of the dimeric SARS-CoV-2 main protease, determined by molecular dynamics simulation. Scientific reports, v. 10, n. 1, p. 1-11, 2020.

26. Chaudhary KK, Mishra N. A review on molecular docking: novel tool for drug discovery. databases, v. 3, n. 4, p. 1029, 2016.

27. Santos-filho OA. Identification of Potential Inhibitors of Severe Acute Respiratory Syndrome-Related Coronavirus 2 (SARS-CoV-2) Main Protease from Non-Natural and Natural Sources: A Molecular Docking Study. Journal of the Brazilian Chemical Society, v. 31, p. 2638$2643,2020$.

28. Kumar Y, Singh H, Patel CN. In silico prediction of potential inhibitors for the main protease of SARS-CoV-2 using molecular docking and dynamics simulation based drugrepurposing. Journal of infection and public health, v. 13, n. 9, p. 1210-1223, 2020.

29. Narkhede RR, et al. The molecular docking study of potential drug candidates showing antiCOVID-19 activity by exploring of therapeutic targets of SARS-CoV-2. Eurasian Journal of Medicine and Oncology, v. 4, n. 3, p. 185-195, 2020.

30. Yu R, et al. Computational screening of antagonists against the SARS-CoV-2 (COVID-19) coronavirus by molecular docking. International Journal of Antimicrobial Agents, v. 56, n. 2, p. 106012, 2020. 